Heck and Suzuki coupling facilitated by $\mathrm{Pd}$ on amine terminated $\mathrm{NiFe}_{2} \mathrm{O}_{4}$ : $\mathrm{A}$ magnetically separable catalyst

Babita Baruwati, Debanjan Guin, Sunkara V Manorama*

Nanomaterials Laboratory, Inorganic and Physical Chemistry Division, Indian Institute of Chemical Technology, Hyderabad 500007

manorama@iict.res.in

Synthesis Procedure \& Characterization techniques

S2-S3

FTIR spectra

S4

XPS and VSM plots

S5

GCMS spectra

S6-S15 
Synthesis of $\mathrm{NiFe}_{2} \mathrm{O}_{4}$ : In brief, stoichiometric amounts of iron (III) nitrate and nickel (II) nitrate were dissolved in deionized water. The $\mathrm{pH}$ of the solution was adjusted to 8 by addition of aqueous ammonia, stirred vigorously for $2 \mathrm{~h}$ and transferred to an autoclave whose temperature was gradually raised to $225^{\circ} \mathrm{C}$ over a period of $2 \mathrm{hrs}$ and maintained there for $2 \mathrm{hrs}$ under auto generated pressure of $20 \mathrm{~kg} / \mathrm{cm}^{2}$. After cooling, the precipitate was filtered, washed, and dried.

Preparation of surface modified nanocrystalline ferrites: $1 \mathrm{~g} \mathrm{NiFe} \mathrm{O}_{4}$ was taken in deionized water. $2 \mathrm{~g}$ of dopamine was added to the mixture and refluxed for 12 hrs at $100^{\circ} \mathrm{C}$. The resulting product was precipitated using excess amount of acetone and centrifugation. It was then dried and used for Pd incorporation.

Incorporation of $\boldsymbol{P d}(0): 1 \mathrm{~g}$ of surface modifided $\mathrm{NiFe}_{2} \mathrm{O}_{4}$ nanoparticle was dispersed in water. $\mathrm{NaPdCl}_{4}$ solution in water was added to the mixture to get 10 $\mathrm{wt} \%$ of $\mathrm{Pd}$. Diluted solution of hydrazine monohydrate was added dropwise to adjust the $\mathrm{pH}$ to 9 . The reaction mixture was stirred overnight at room temperature and then allowed to settle. The product was washed several times with water, centrifuged and dried at room temperature.

Henceforth the catalyst is referred as $\mathrm{NiFe}_{2} \mathrm{O}_{4}$-DA-Pd.

\section{Characterization Techniques}

X-Ray diffraction patterns of the powdered samples are recorded on a Rigaku Miniflex Table Top diffractometer using CuK $\alpha$ radiation $\left(1.5406 A^{\circ}\right)$ source. The intensity data were collected over a $2 \theta$ range of 2 to 80 using a step scan mode ( 0.06 degree per sec). The average crystallite size of the samples was estimated with the help of Scherrer equation using the FWHM applied to all major peaks.

The amount of $\mathrm{Pd}$ in the samples before and after reactions are determined with an IRIS Intrepid II XDL inductively coupled plasma, atomic emission spectroscopy (ICPAES) instrument. $2.5 \mathrm{mg}$ of each sample was dissolved in $2.5 \mathrm{~mL}$ of concentrated aqua regia and the volume was adjusted to $25 \mathrm{~mL}$. $5 \mathrm{~mL}$ of this solution was then further diluted to $25 \mathrm{~mL}$. This diluted sample was then used for the AES analysis.

Transmission electron micrographs were recorded on a Phillips Tecnai $G^{2}$ FEI F12 electron microscope. The powder samples are dispersed in ethanol by ultrasonication before loading onto a carbon coated copper grid and then allowed to dry at room temperature before recording the micrographs.

FTIR spectra were recorded on a Nicolet Nexus 670 Spectrometer equipped with DTGS KBr detector in the range $400-4000 \mathrm{~cm}^{-1}$.

XPS measurements for the as synthesized samples were performed on a Kratos X-Ray photoelectron spectrometer. The X-ray excitation energy was $1253.6 \mathrm{eV}(\mathrm{MgK} \alpha)$ and the spectra were recorded with pass energy of $80 \mathrm{eV}$. The 
angle between the detector and the X-ray flux direction was constant and equal to $90^{\circ}$, while the measurements were made at an electron take off angle (angle between the surface plane and electron emission direction) of $70^{\circ}$. The calibration of the spectrometer and the charging correction were verified by determining the binding energy of $\mathrm{C}$ 1s level with a graphite substrate (at $285 \mathrm{eV}$ ). The binding energies were compared with the reported data from literature to identify the chemical composition and the oxidation state.

GCMS spectra were recorded on a Thermo Finnigan TRACE DSQ MS Spectrophotometer using BP-1 $(30 \times 0.25 \times 1.0)$ capillary column.

Magnetic hysterisis loop of the pure $\mathrm{NiFe}_{2} \mathrm{O}_{4}$ sample was recorded on a Lakeshore 7000 Vibrating sample magnetometer up to a field 1T. 


\section{S4}

\section{FTIR}

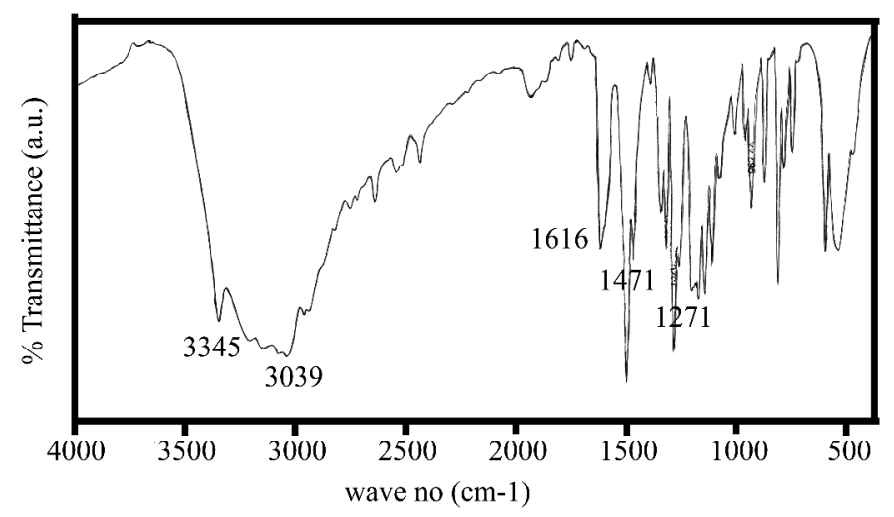

FTIR spectrum of Dopamine

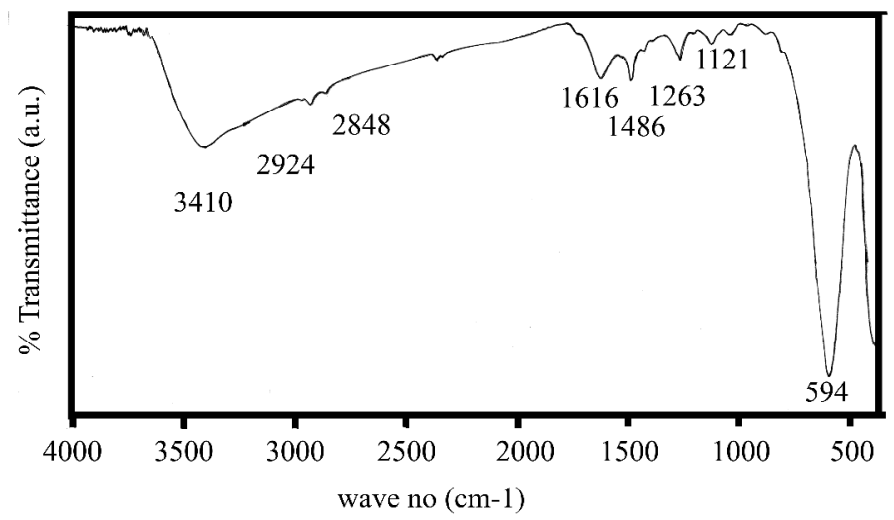

FTIR spectrum of $\mathrm{NiFe}_{2} \mathrm{O}_{4}$ functionalized with dopamine

The peak at $3410 \mathrm{~cm}^{-1}$ has been assigned to the surface adsorbed water and peaks at $2924 \mathrm{~cm}^{-1}$ and $2848 \mathrm{~cm}^{-1}$ are assigned to the $\mathrm{C}-\mathrm{H}$ stretching vibrations of aromatic groups. The peak at $1616 \mathrm{~cm}^{-1}$ is due to the $\mathrm{N}-\mathrm{H}$ bending. The peak at $1486 \mathrm{~cm}^{-1}$ is due to $\mathrm{C}-\mathrm{H}$ bending of aromatic groups while peaks at 1263 and $1121 \mathrm{~cm}^{-1}$ are due to $\mathrm{C}-\mathrm{O}$ stretching and aliphatic $\mathrm{C}-\mathrm{H}$ bending respectively. The sharp peak at $594 \mathrm{~cm}^{-}$ ${ }^{1}$ is the IR signature for ferrite particles. 
XPS

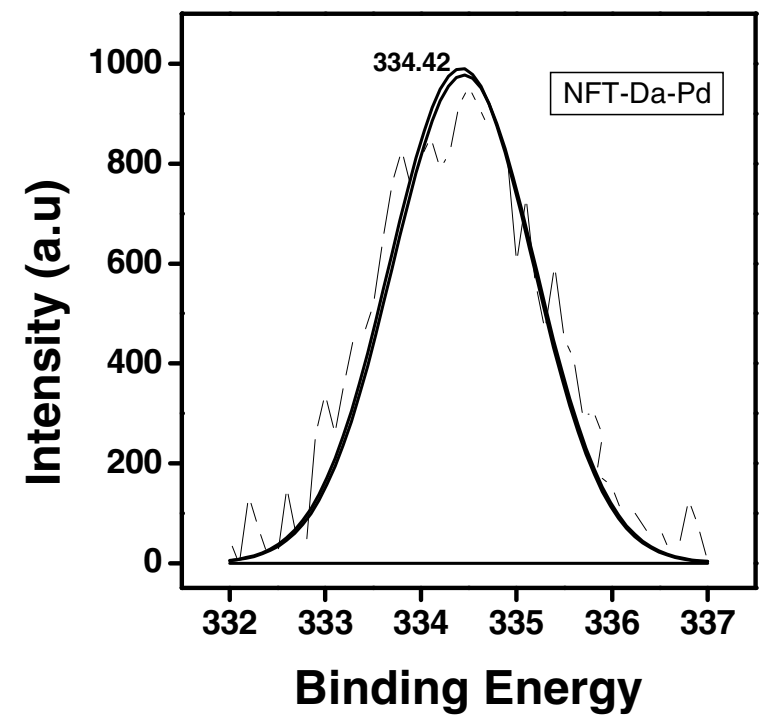

Binding energy spectra of $\mathrm{Pd} 3 \mathrm{~d} 5 / 2$ in the as synthesized catalyst showing the presence of only $\mathrm{Pd}(0)$ in it.

\section{VSM}

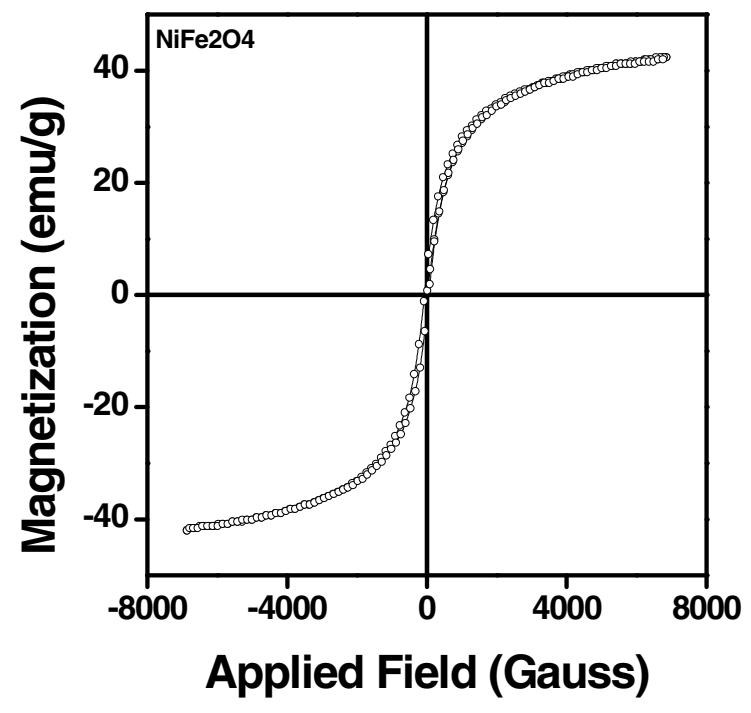

Magnetization Vs Applied field plot for the as synthesized $\mathrm{NiFe}_{2} \mathrm{O}_{4}$ nanoparticles showing the superparamagnetic behavior. 


\section{S6}

\section{GCMS}

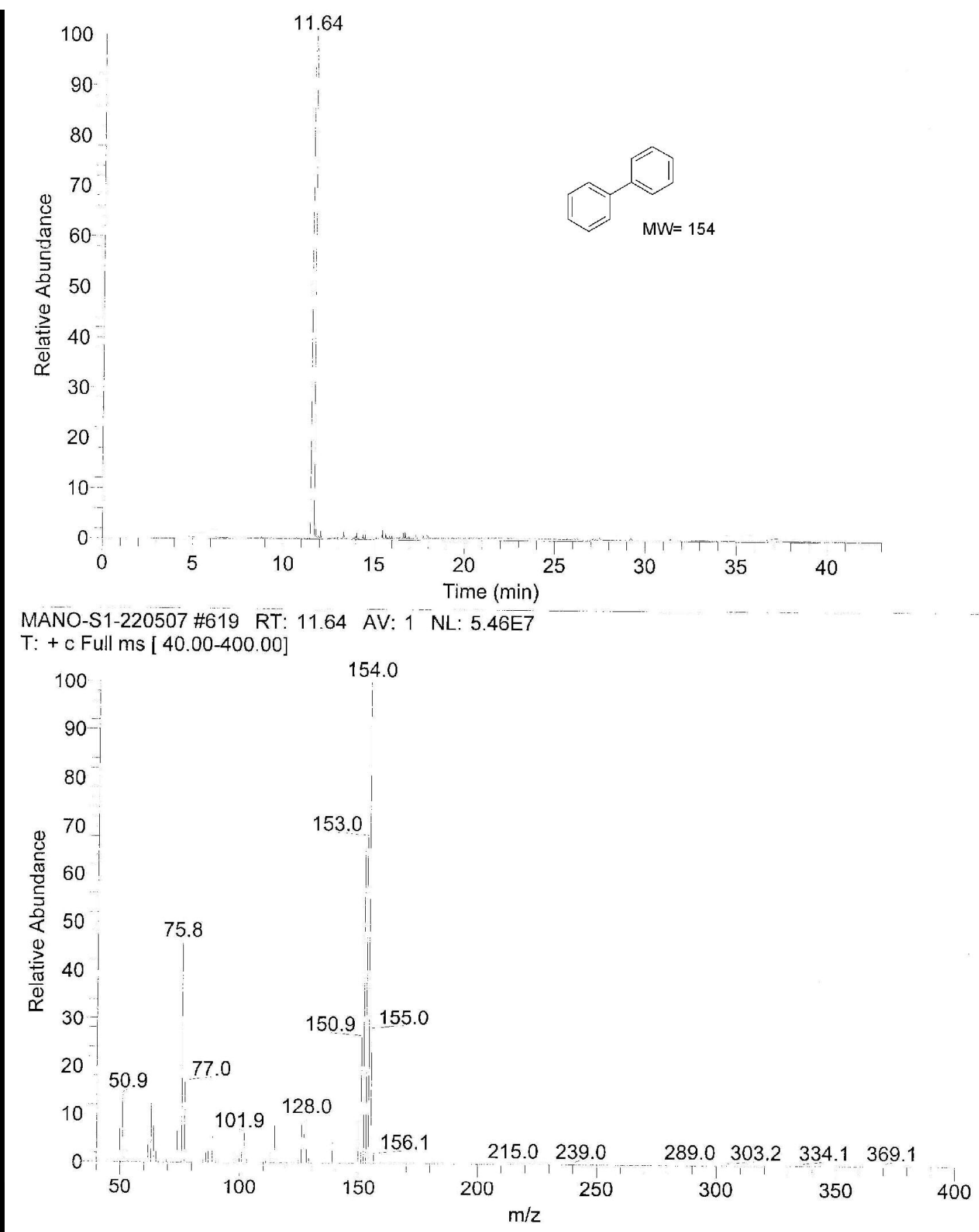




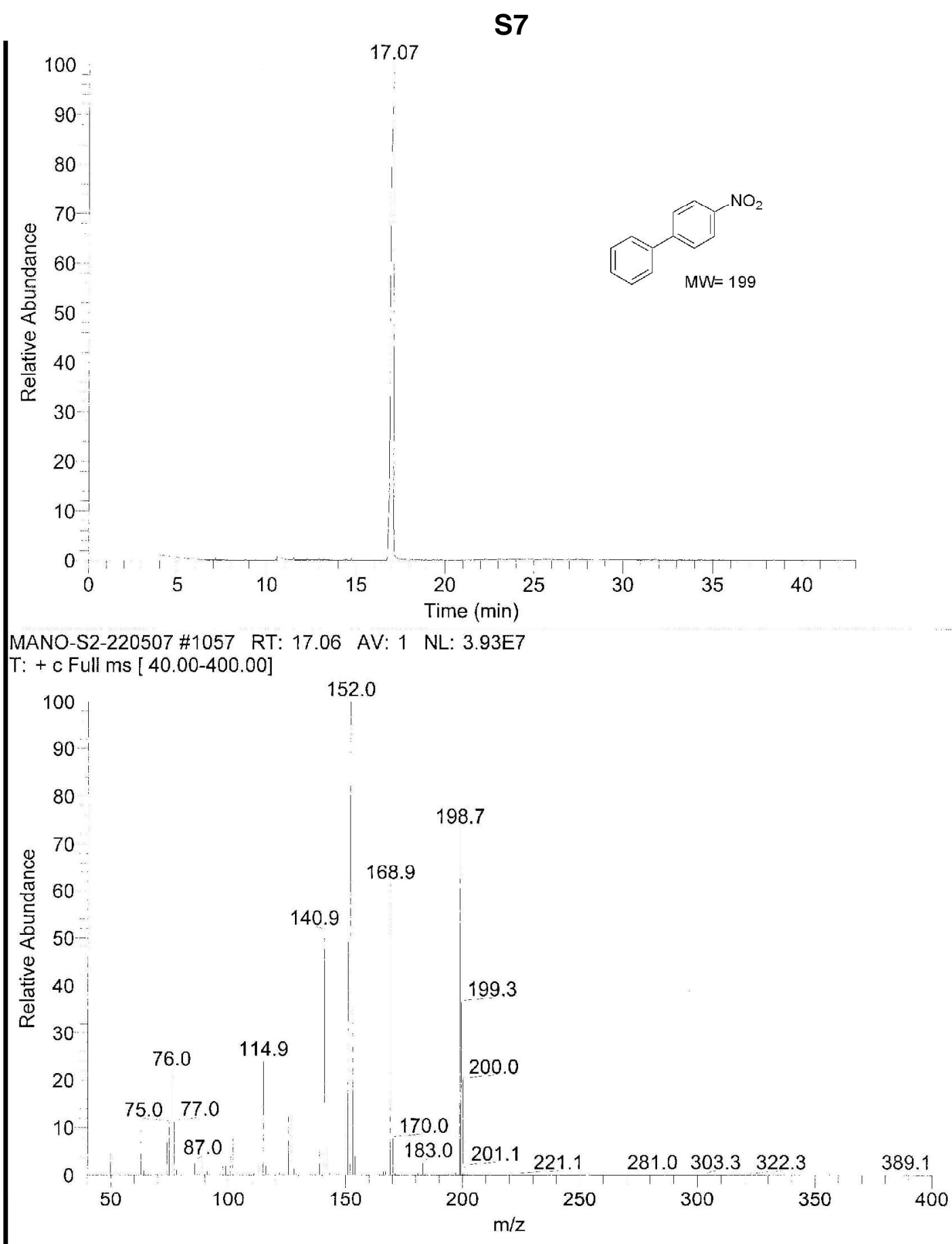




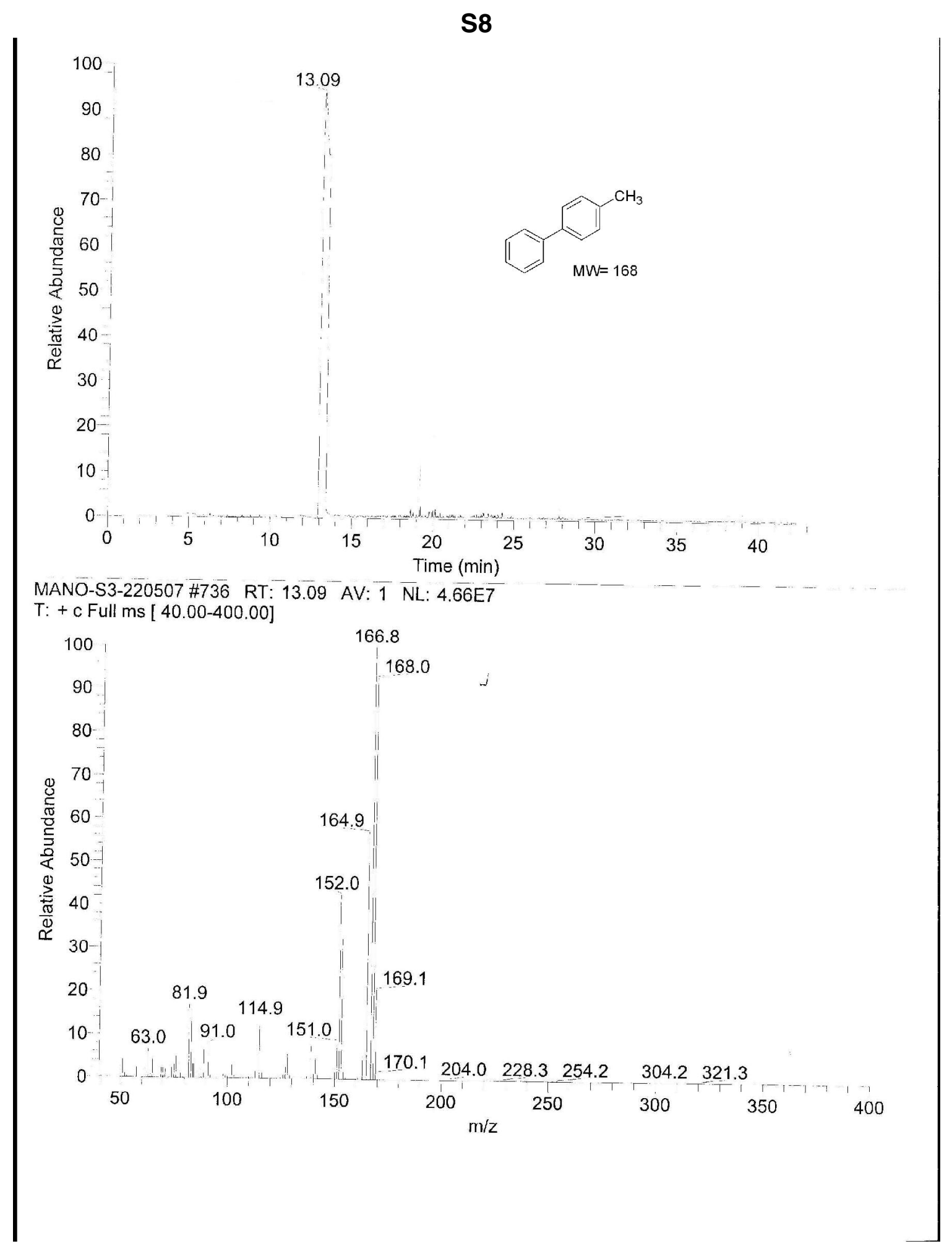




\section{S9}

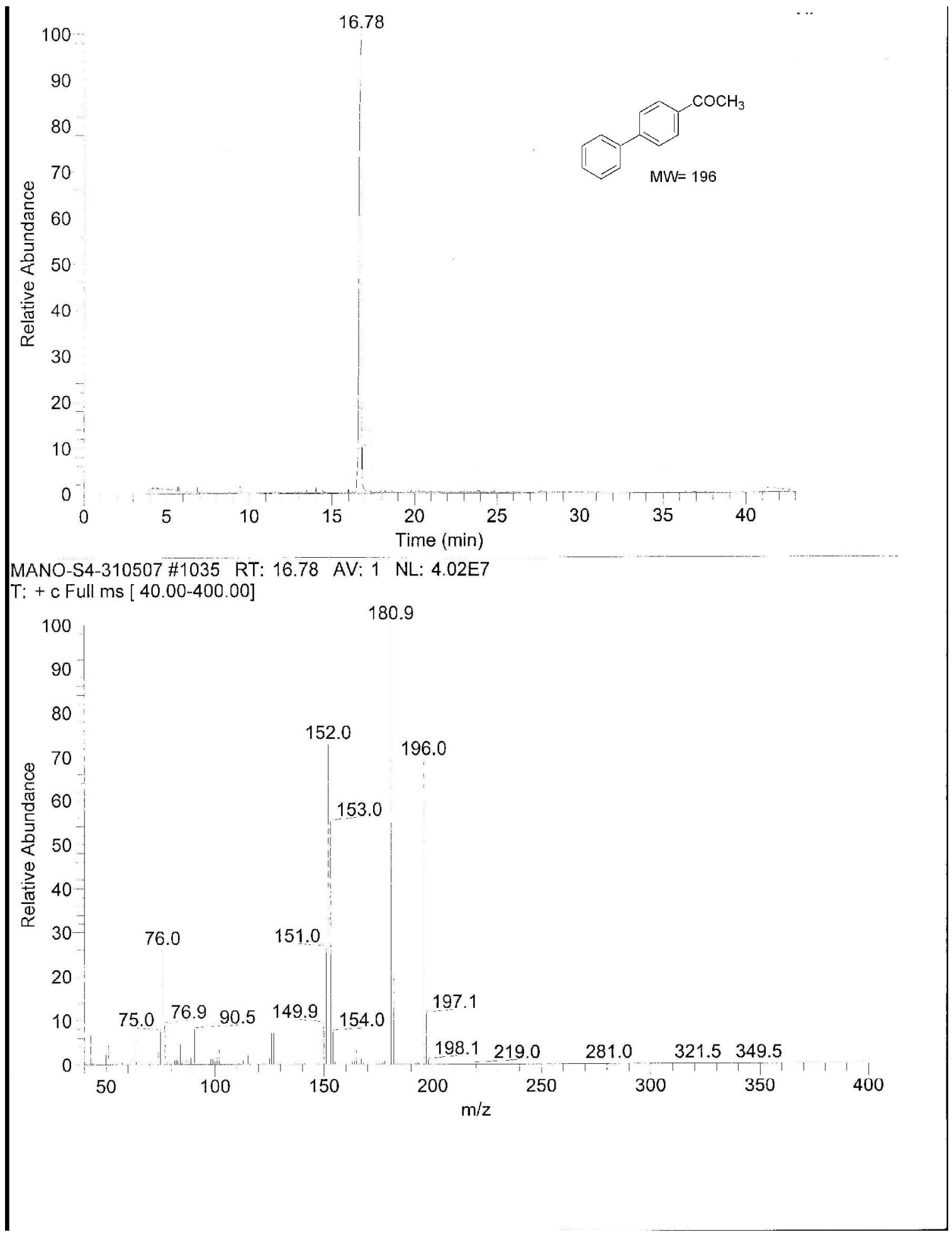




\section{$\mathrm{S} 10$}

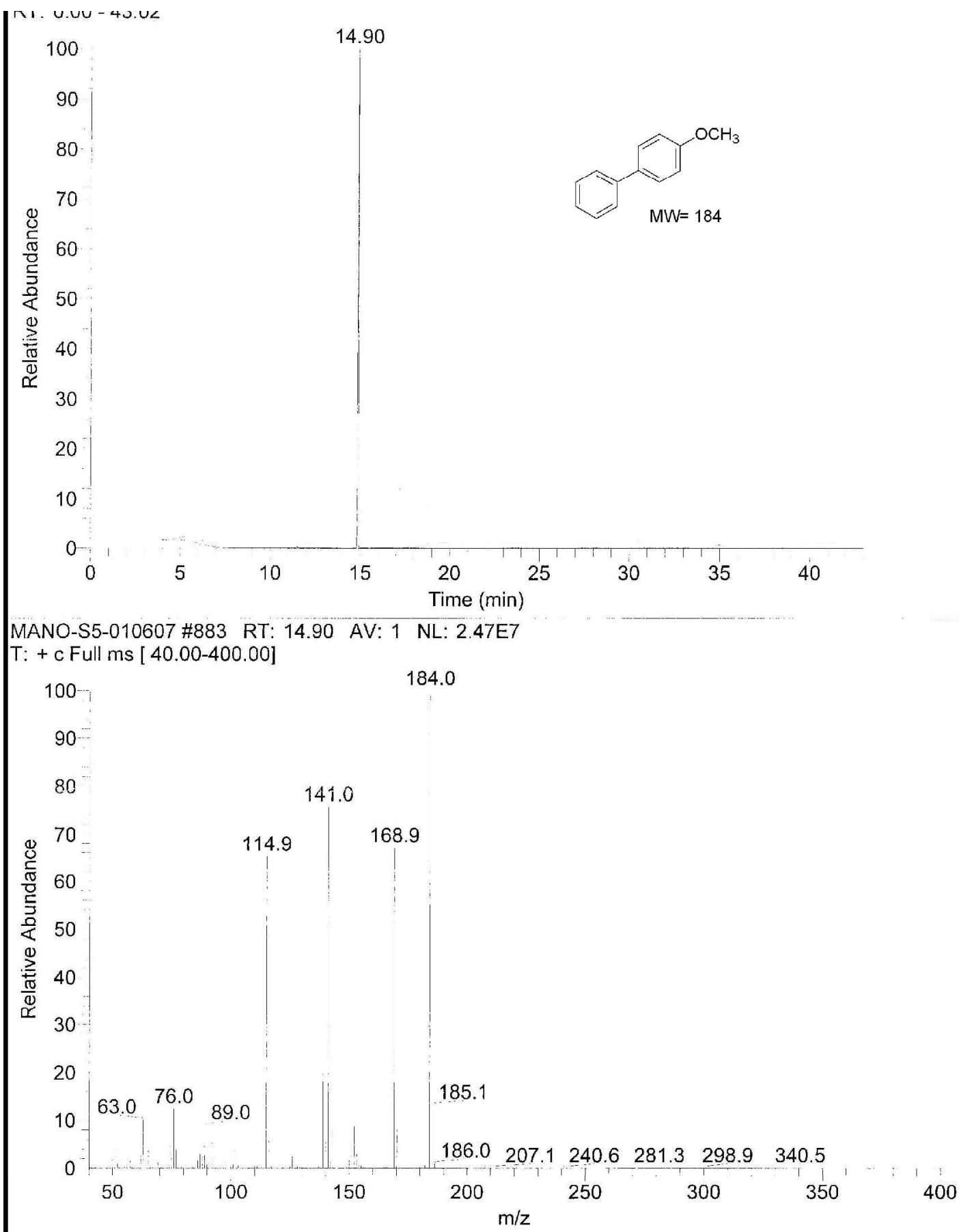




\section{S11}

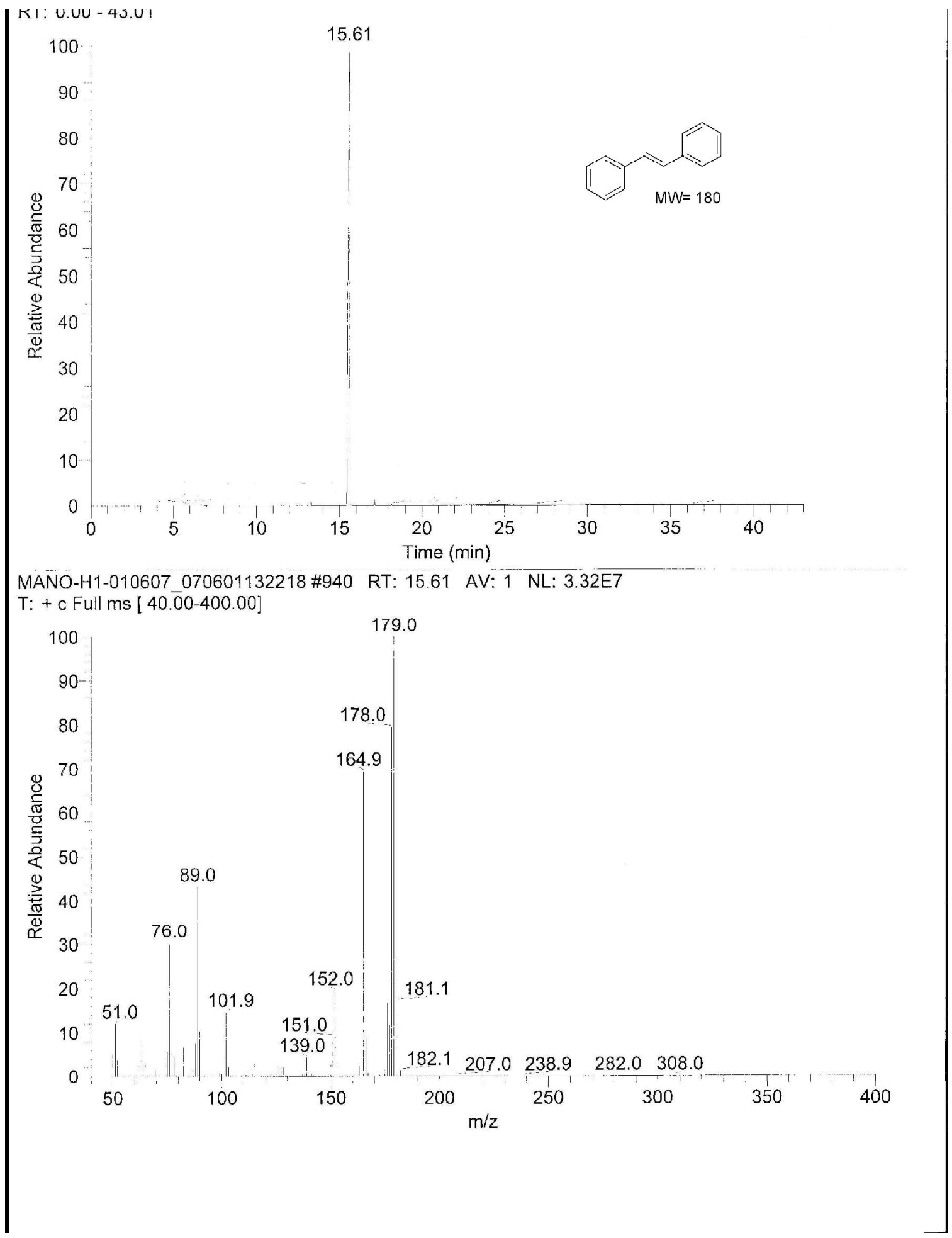




\section{S12}

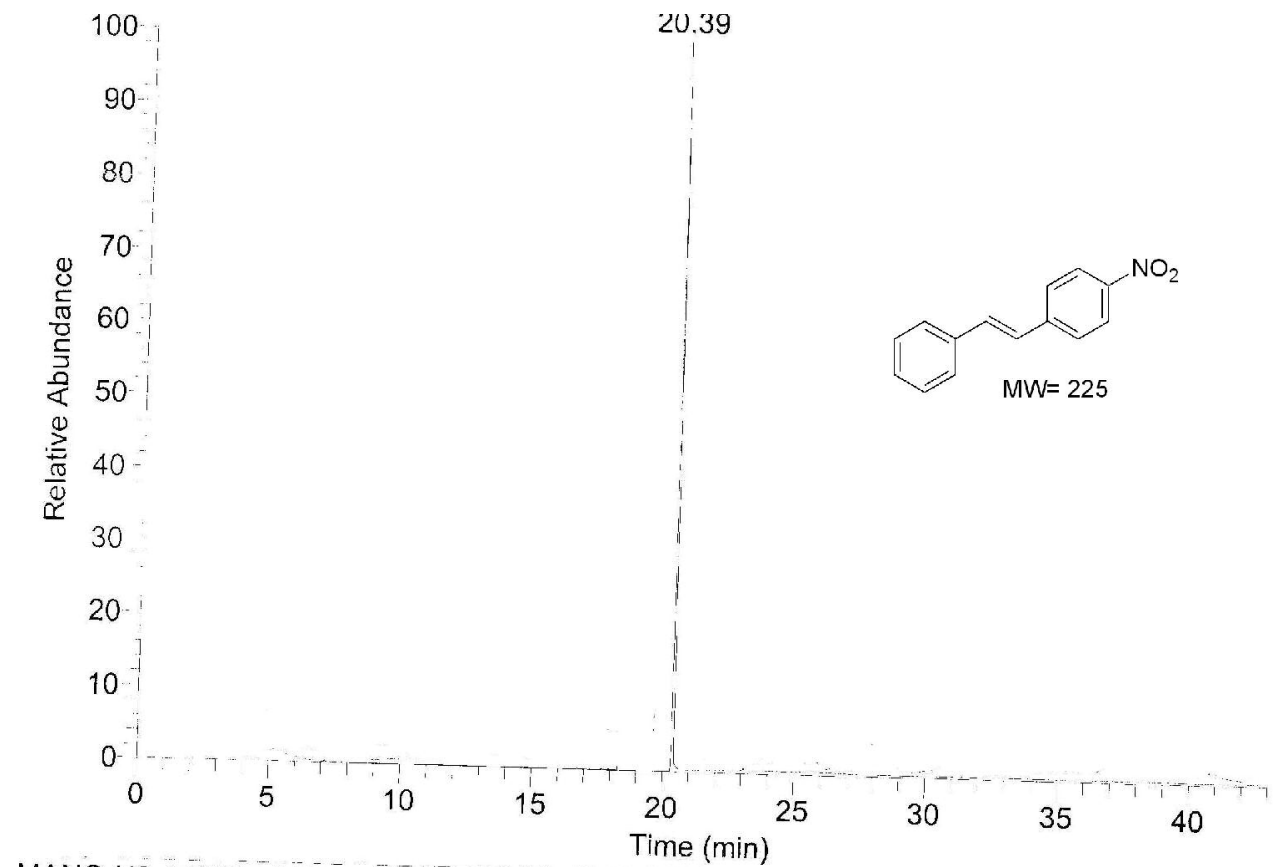

MANO-H2-170507 \#1327 RT: $20.3 \overline{9}$ AV: 1 NL: $2.2 \overline{6} \overline{\mathrm{E}}$

$\mathrm{T}:$ + c Full ms [ 40.00-400.00]

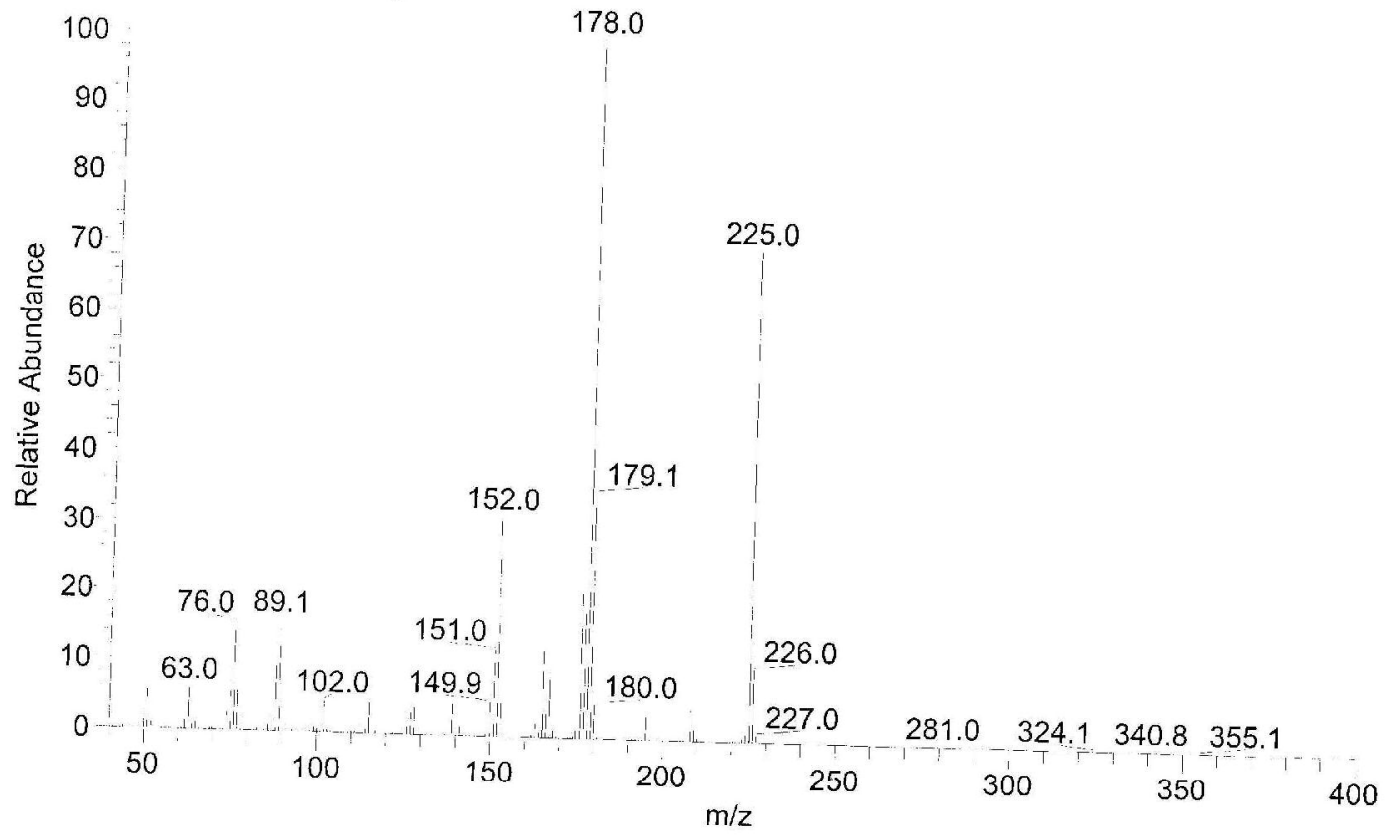




\section{S13}

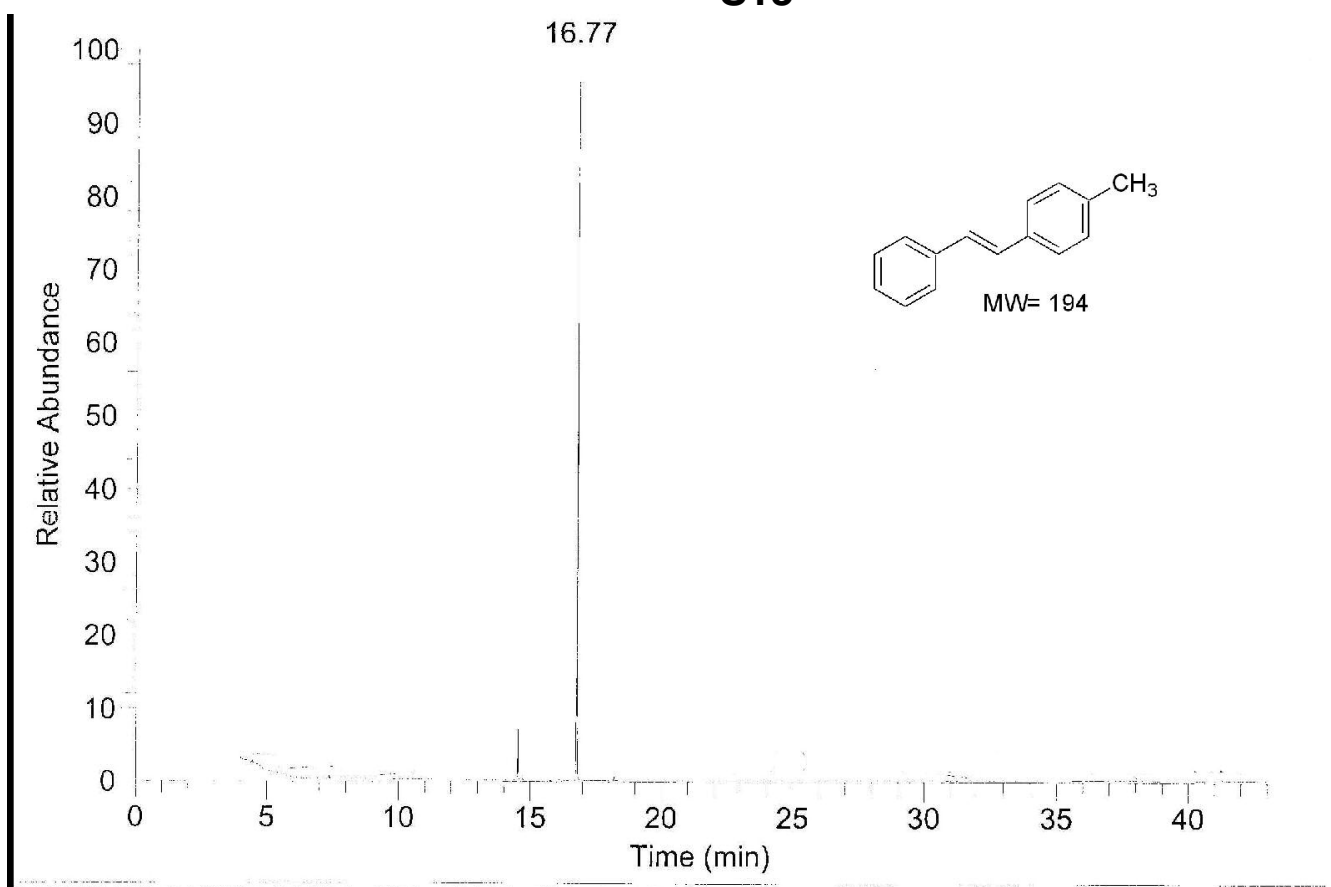

MANO-H3-310507 \#1034 RT: 16.77 AV: 1 NL: 1.16E7 $\mathrm{T}:+\mathrm{c}$ Full ms [ 40.00-400.00]

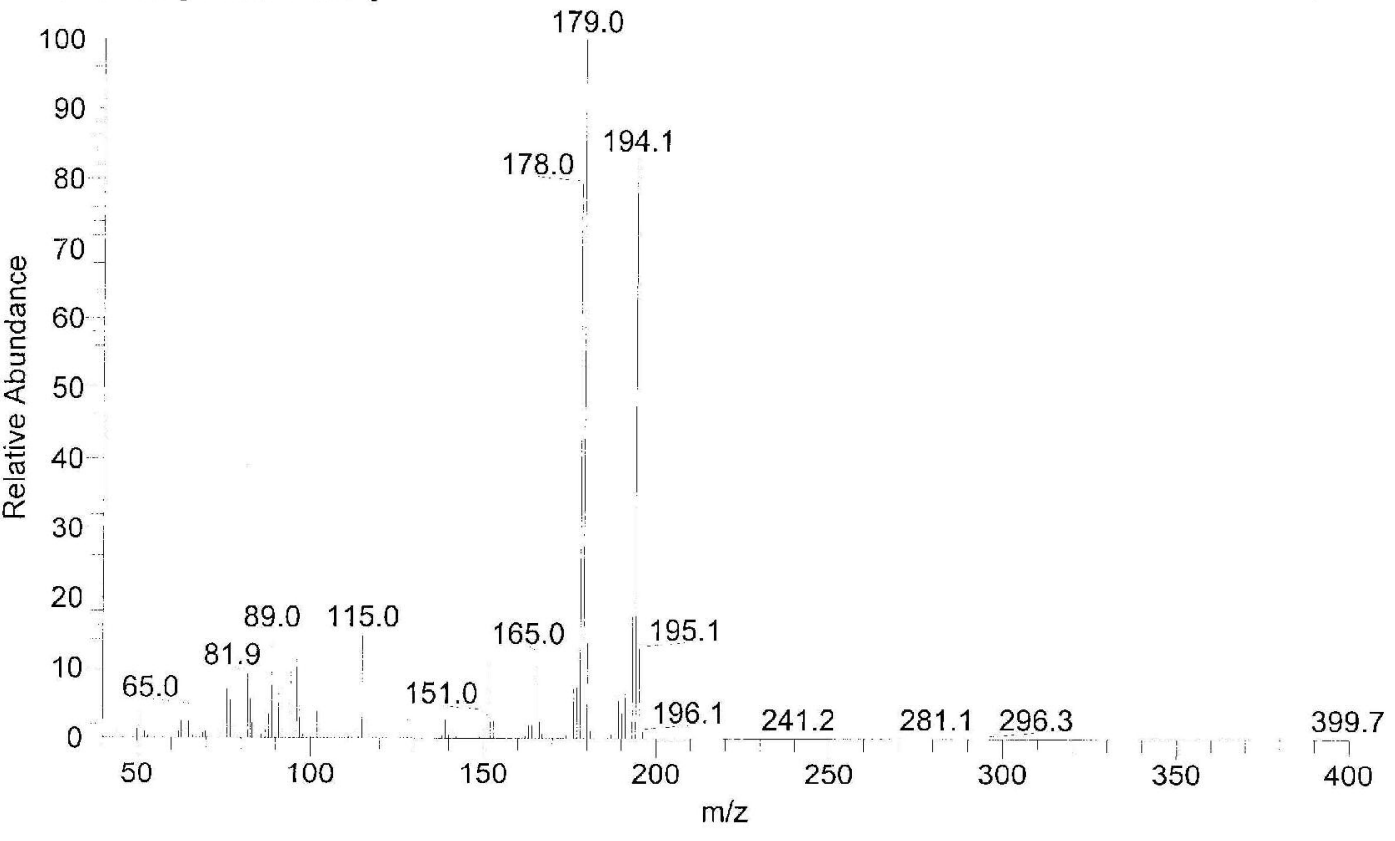




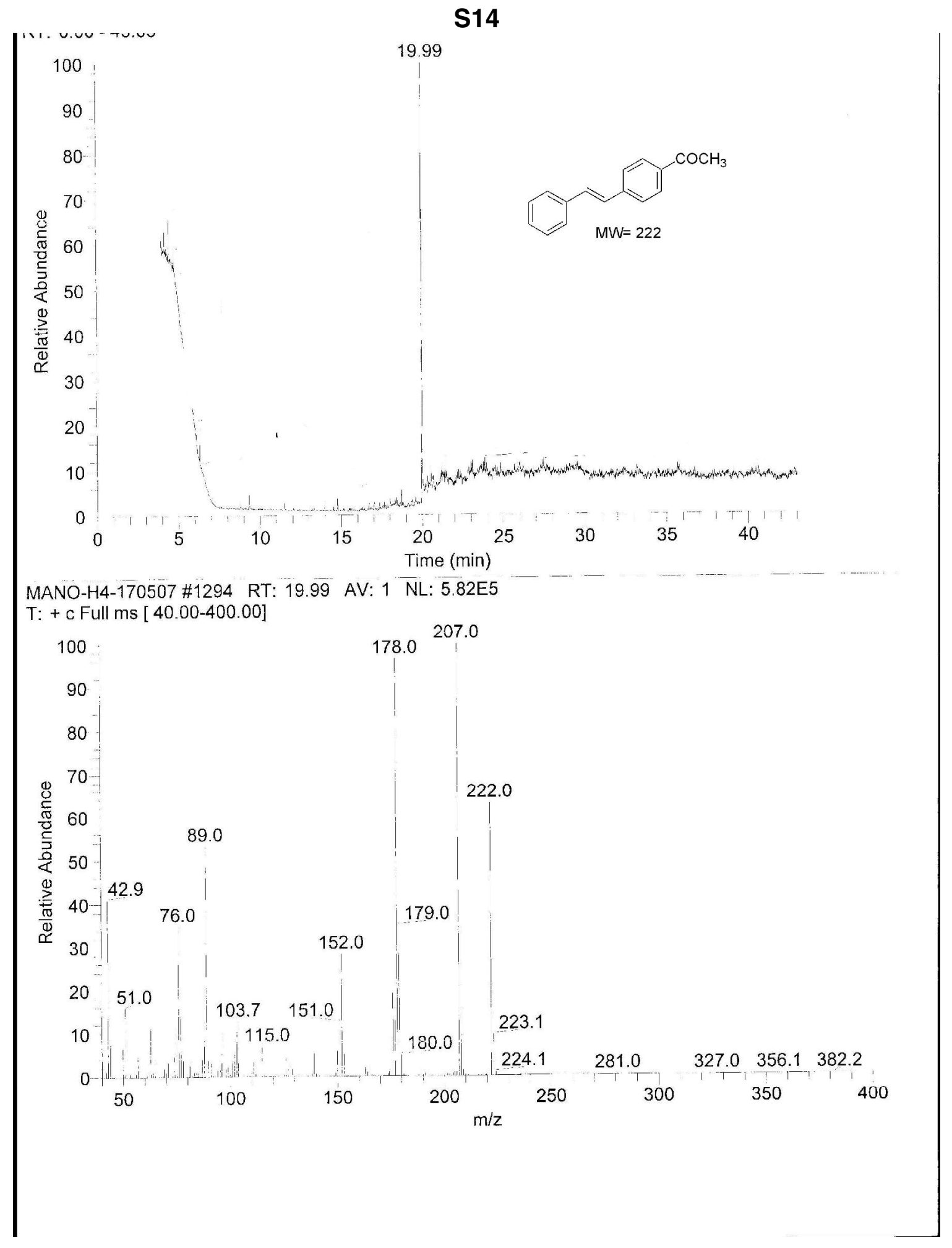




\section{S15}

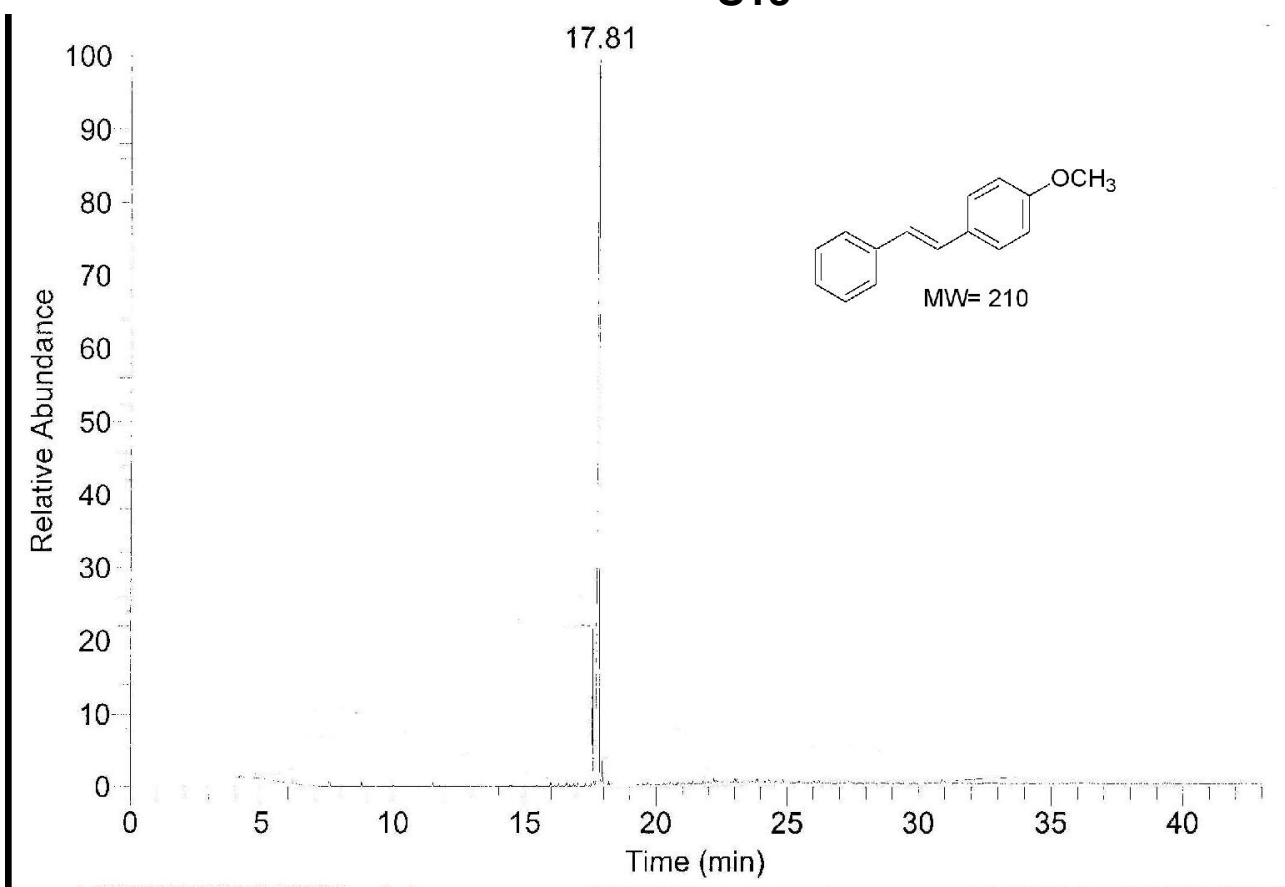

MANO-H5-180507 \#1118 RT: 17.81 AV: 1 NL: 4.04E7

T: + c Full ms [ 40.00-400.00]

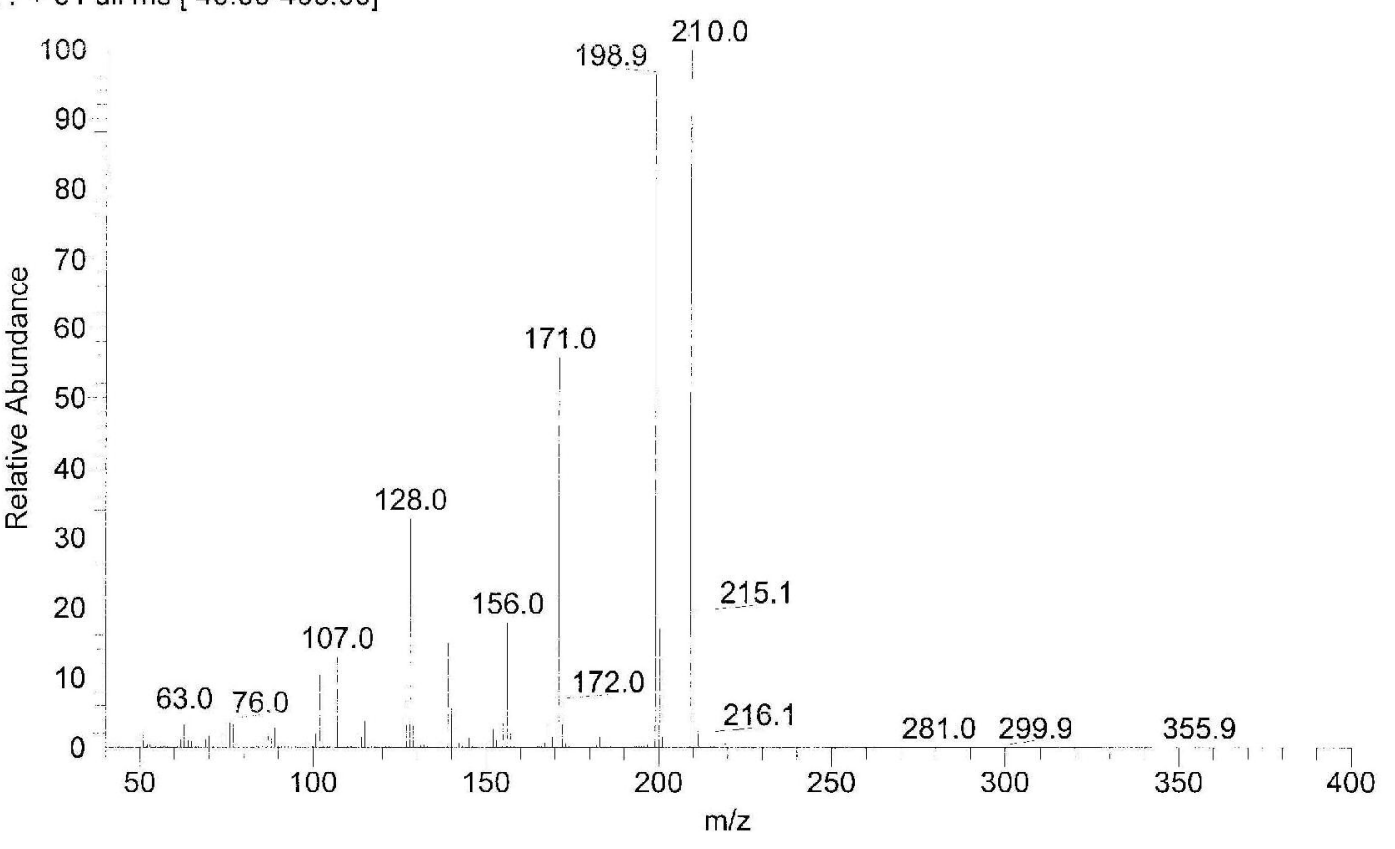

